\title{
Crossover from superfluidity to superconductivity in a system with doping dependent attraction
}

\author{
V.M. Loktevaand V. Turkowski ${ }^{\mathrm{b}} *$

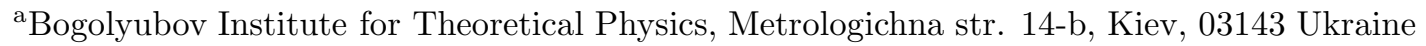 \\ ${ }^{\mathrm{b}}$ CFIF, Instituto Superior Tecnico, Av.Rovisco Pais, 1049-001 Lisbon, Portugal
}

\begin{abstract}
Zero temperature crossover from superfluidity to superconductivity with carrier density increasing is studied for a two-dimensional system in the $s$-wave and $d$-wave pairing channels. It was assumed that the particle attraction correlation length depends on carrier density $n_{f}$ as $r_{0} \sim 1 / \sqrt{n_{f}}$. Such a dependence was found experimentally for the radius of magnetic correlations in $\mathrm{La}_{2-x} \mathrm{Sr}_{x} \mathrm{CuO}_{4}$. The short range Coulomb repulsion was also taken into account. It is shown that the behavior of the system with doping is fundamentally different from the case with $r_{0}\left(n_{f}\right)=$ const. In particular, similarly to the $d$-wave case, the crossover in the $s$-channel takes place only if the coupling is larger of some minimal value, otherwise the Cooper pairing scenario takes place at any small carrier density. The relevance of the model to the high-temperature superconductors is discussed.
\end{abstract}

PACS : 74.20.-z, 74.62.Dh, 74.72.-h

Keywords : Symmetry, $s$-Wave, $d$-Wave, Effects of Doping

\section{INTRODUCTION}

The problem of the crossover from superfluidity to superconductivity with charge carrier density or coupling constant changing has a long history [1]. The interest to this phenomena has arisen again after the discovery of high-temperature superconductors (HTSCs) in 1986 [2 10]. It was already known upon that time that the superfluidity of composite bosons transforms into the superconductivity of overlapped Cooper pairs with chemical doping in the case of the s-wave pairing. Now, in the s-wave pairing case the problem is quite well explored for the 3D systems [2 4 ] and, particularly for the quasi-2D case 5 ]. For the $2 \mathrm{D}$ case this problem was studied at $T=0$ ( when a long-range superconducting is still possible in a 2D system [11]) for the case of local attraction (see, for example [2, 3.,6]) and for the phonon-exchange model [7]. Most of these problems are reviewed in [12].

Due to a layered structure and the anisotropic symmetry of the order parameter in HTSCs, the

\footnotetext{
*Corresponding author.Tel.: +351-21-8419094; fax: +351-21-8419143. E-mail address: vturk@cfif.ist.utl.pt
}

crossover in the $2 \mathrm{D}$ system in the $d$-wave pairing channel is of a special interest. However, because if its complexity, this case is not so well understood at present. The d-wave pairing for the case of the extended Hubbard model with the nearest neighbor attraction case was analyzed in [8,9. The crossover from superfluidity to superconductivity in the $s$-wave and $d$-wave pairing channels for a $2 \mathrm{D}$ continuum model was studied in paper [10], where also thermodynamic properties of the system in the crossover particle density region were considered. The authors proposed an interaction potential, which is attractive at distances between particles shorter of some value $r_{0}$ and longer of $r_{1}$, and repulsive due to electron correlations at short distances, $r<r_{1}$. It was found, in particular, that there exists a minimal value of the attractive coupling constant in the $d$-wave pairing channel, which gives the crossover from superfluidity to superconductivity at small carrier densities, i.e. the fermion chemical potential changes its sign and becomes positive with carrier density growth.

The correlation length $r_{0}$ was assumed in 10 to be a parameter, which does not depend on the 
carrier density $n_{f}$ per cell. However, concerning HTSCs it would be interesting to consider a more realistic case, when at small carrier densities $r_{0} \sim 1 / \sqrt{n_{f}}$ and the proportionality coefficient is of order of a lattice constant. Such a dependence was experimentally observed in HTSC cuprates for the length of spin-spin correlations, which are believed to be responsible for the hole attraction in these materials. In particular, it was found for $\mathrm{La}_{2-x} \mathrm{Sr}_{x} \mathrm{CuO} \mathrm{O}_{4}$ in underdoped regime, that the magnetic correlation length decreases with carrier density per cell according to the dependence $3.8 \AA / \sqrt{n_{f}} 113$.

In what follows we analyze the possibility of low carrier density crossover for a model analogous to [10] with particle repulsion at distances $r<r_{1}$ and attraction at $r_{1}<r<r_{0}$, where, however,

$r_{0}=\frac{a}{\sqrt{n_{f}}}$

( $a$ is parameter of order of the lattice constant, and its possible value is discussed in the next Section). Obviously, at large carrier densities such that $r_{1}>r_{0}$ the superconductivity in this system should disappear. However, since we are interested in the small carrier concentrations, it is assumed that this relation does not take place.

As it will be shown below such a dependence of $r_{0}$ leads to a qualitatively different behavior of the system with respect to the case with $r_{0}\left(n_{f}\right)=$ const. In particular, there exists a minimal value for the coupling constant when the twoparticle bound states exist at low carrier densities in the $s$-wave pairing channel. The existence of such a threshold value of the coupling constant is typical for the $d$-wave pairing case (see, for example 10,14). Another interesting property is: for any coupling constant in both channels there exists a corresponding carrier density value, below which the system is in the superconducting state.

\section{THE MODEL AND THE MAIN EQUATIONS}

The Hamiltonian of the system which describes the non-retarded fermion interaction can be writ- ten in a standard form

$$
\begin{array}{r}
H=-\sum_{\sigma=\uparrow, \downarrow} \int d x \psi_{\sigma}^{\dagger}(x)\left(\frac{\nabla^{2}}{2 m}+\mu\right) \psi_{\sigma}(x)+ \\
\iint d x_{1} d x_{2} \tilde{V}\left(x_{1}, x_{2}\right) \psi_{\uparrow}^{\dagger}\left(x_{1}\right) \psi_{\downarrow}^{\dagger}\left(x_{2}\right) \psi_{\downarrow}\left(x_{2}\right) \psi_{\uparrow}\left(x_{1}\right),
\end{array}
$$

where $m$ is the effective fermion mass, and $\mu$ is the chemical potential; fermi-operators $\psi_{\sigma}(x)$ depend on the space-time coordinate $x=(\mathbf{x}, t)$. The instantaneous interaction potential is chosen in the next form

$$
\tilde{V}\left(x_{1}, x_{2}\right)=\delta\left(t_{1}-t_{2}\right) V(r),
$$

with

$V(r)=V_{\text {rep }} \theta\left(r_{1}-r\right)-V_{a t t r} \theta\left(r-r_{1}\right) \theta\left(r_{0}-r\right)$,

which corresponds to potential used in [10]. Here $r=\left|\mathbf{x}_{1}-\mathbf{x}_{2}\right|$ is an inter-particle distance. Positive parameters $V_{\text {rep }}$ and $V_{\text {attr }}$ correspond to particle repulsion at $r<r_{1}$ and particle attraction at $r_{1}<r<r_{0}$. The charge carrier density dependence of the correlation radius $r_{0}$ is given by (1) with the parameter $a=\sqrt{2 / \pi} a_{0}, a_{0}$ is the square lattice constant. This relation can be easily estimated from the equality $(\pi / 2) r_{0}^{2} N_{f}=a_{0}^{2} N_{\text {cell }}$, where on the left side the volume of the $2 \mathrm{D}$ system is expressed as a volume (circle of the radius $\sim r_{0}$ ) occupied by one particle, multiplied by the full number of particles $N_{f}, N^{\text {cell }}$ is an elementary cell number in the system. The free fermion bandwidth $W$ is connected with $a_{0}$ as $W=\pi^{2} /\left(m a_{0}^{2}\right)$. It should be noted, that the relation (1) at $a=\sqrt{2 / \pi} a_{0}$ is in a good agreement with the experimental data for $\mathrm{La}_{2-x} \mathrm{Sr}_{x} \mathrm{CuO}$ [13], where the plane magnetically ordered lattice parameters are equal to $5.354 \AA$ and $5.401 \AA$, and the corresponding parameter $a$ is $\simeq 3.8 \AA$.

In order to study the superconducting properties of the model in the channels with different pair angular momentum $l$, it is convenient, similarly to [10], to approximate the Fourier transform of (3) by a separable potential:

$V_{\mathbf{k}_{1} \mathbf{k}_{2}}^{l}=-\lambda_{l} w_{l}\left(\mathbf{k}_{1}\right) w_{l}\left(\mathbf{k}_{2}\right)$,

where $\lambda_{l}$ is an effective coupling constant, and functions

$w_{l}(\mathbf{k})=h_{l}(k) \cos \left(l \varphi_{\mathbf{k}}\right)$ 
with coefficients

$h_{l}(k)=\frac{\left(k / k_{1}\right)^{l}}{\left(1+k / k_{0}\right)^{l+1 / 2}}$,

$k$ is the momentum modulus $k=|\mathbf{k}|$, and $\varphi_{\mathbf{k}}$ is its angle in polar coordinates $\mathbf{k}=$ $\left(k \cos \left(\varphi_{\mathbf{k}}\right), k \sin \left(\varphi_{\mathbf{k}}\right)\right)$. Parameters $k_{0}$ and $k_{1}$ put the momentum range in the proper region. They are connected with the potential (3) parameters as $k_{0} \sim 1 / r_{0}$ and $k_{1} \sim 1 / r_{1}$. Below we put $k_{0}=1 / r_{0}$ and $k_{1}=1 / r_{1}$. Obviously, the expression (⿶) is not the exact Fourier transform of (3), but it sets the interaction in right momentum range and has the correct asymptotic behavior at small and large momenta: $V_{\mathbf{k}_{1} \mathbf{k}_{2}}^{l} \sim k_{1}^{l} k_{2}^{l}$ and $V_{\mathbf{k}_{1} \mathbf{k}_{2}}^{l} \sim 1 / \sqrt{k_{1} k_{2}}$, correspondingly. Since we are mainly interested in the low carrier density region, where the crossover can take place, the correct behavior of the interaction potential at small momenta is the most important. In the case of low carrier concentrations the small momenta give the main contribution in the integral of the gap equation (see Eq. (7) below). We shall study $s$ - and $d$-wave channels with $l=0$ and 2 separately, so we assume that the parameters $\lambda_{l}$ for both channels are independent. It will be assumed also that the pairing takes place at zero pair momentum at $T=0$.

The minimization of the ground state energy with respect to the superconducting order parameter $\Delta_{l}\left(x_{1}-x_{2}\right)=V\left(x_{1}-x_{2}\right)<\psi_{\uparrow}\left(x_{1}\right) \psi_{\downarrow}\left(x_{2}\right)>$ and the chemical potential gives in the case of the approximation (4) the standard pair of equation for the crossover problem (see, for example [10]):

$$
\begin{aligned}
& \Delta_{l}(\mathbf{k})=-\lambda_{l} \int \frac{d \mathbf{p}}{(2 \pi)^{2}} \frac{\Delta_{l}(\mathbf{p})}{2 \sqrt{\varepsilon^{2}(\mathbf{p})+\Delta_{l}(\mathbf{p})^{2}}} V_{\mathbf{p}, \mathbf{k}}^{l}, \\
& \int \frac{d \mathbf{p}}{(2 \pi)^{2}}\left(1-\frac{\varepsilon(\mathbf{p})}{\sqrt{\varepsilon^{2}(\mathbf{p})+\Delta_{l}(\mathbf{p})^{2}}}\right)=\frac{n_{f}}{a_{0}^{2}}
\end{aligned}
$$

where $\varepsilon(\mathbf{p})=\mathbf{p}^{2} / 2 m-\mu$ is a free particle dispersion law. The form of the equation (7) allows to search the solution for the superconducting order parameter in the next form

$\Delta_{l}(\mathbf{k})=\Delta_{0 l} w_{l}(\mathbf{k})$,

where $\Delta_{0 l}$ does not depend on momentum $\mathbf{k}$ and $w_{l}(\mathbf{k})$ is defined by (5) and (6). In this case the solution of the system (77), (8) gives the dependence
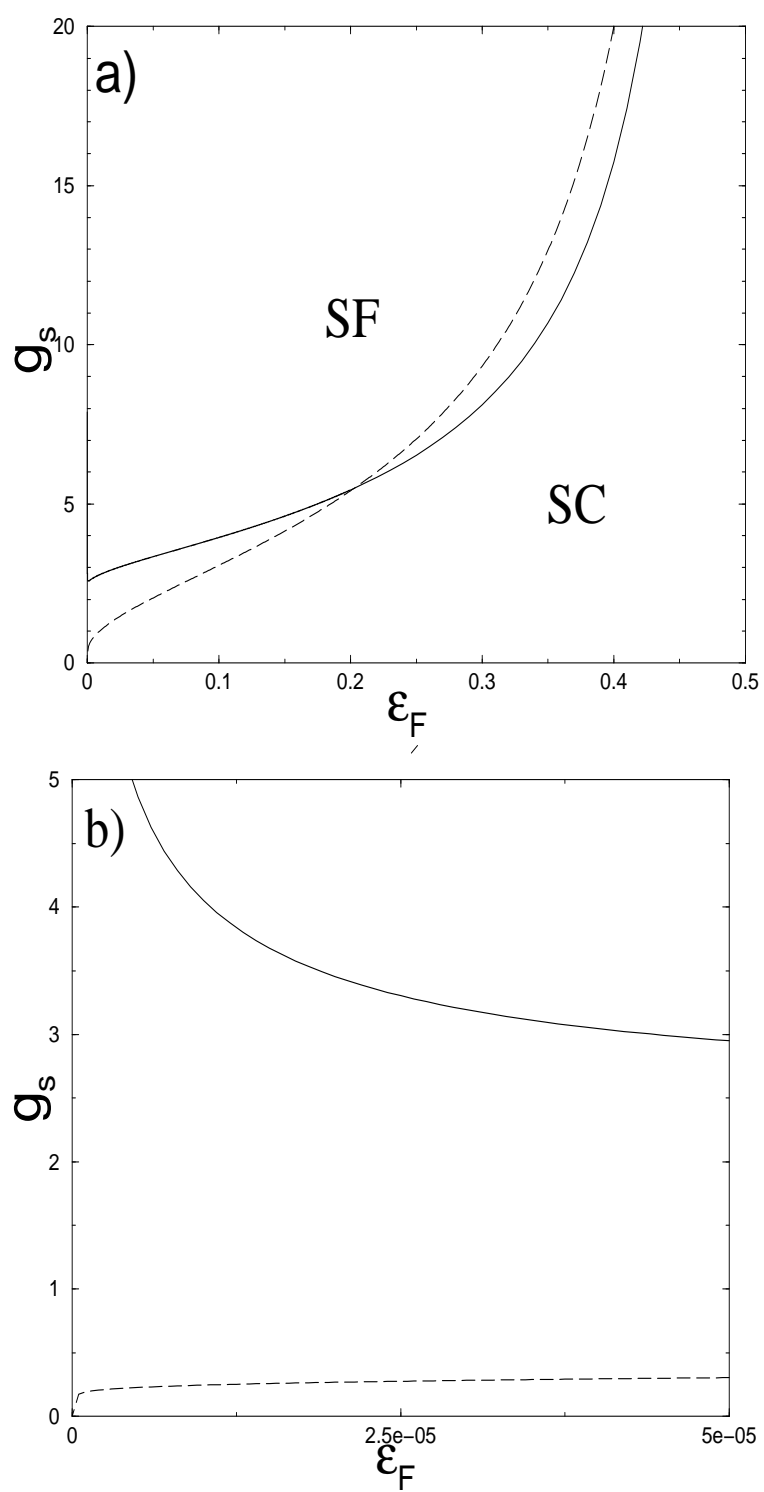

Figure 1. a) Crossover line $g_{s}-\varepsilon_{F}$ is presented for the $s$-pairing channel (solid line). The dotted line represents the corresponding curve for the case with $r_{0}\left(n_{f}\right)=$ const at $r_{0}=a_{0}$. Here and below all parameters are expressed in units of the bandwidth $W$. b) The same as a) at very low charge carrier densities. 
of the gap parameter $\Delta_{0 l}\left(\Delta_{0 s}\right.$ for $l=0$ and $\Delta_{0 d}$ for $l=2$ ) and the corresponding chemical potential $\mu$ on the particle density $n_{f}$ and the coupling $\lambda_{l}$.

\section{CROSSOVER FROM SUPERFLUID- ITY TO SUPERCONDUCTIVITY}

\section{1. $s$-wave pairing channel}

The solution of the system (7), (8) at $\mu=0$ gives the crossover line $\lambda_{s}\left(n_{f}\right)$, which separates the parameters regions, where the local $(\mu<0)$ and Cooper pairing $(\mu>0)$ take place. Note, that in the $s$-wave pairing case the Coulomb repulsion parameter $k_{1}$ does not enter in the equations. The numerical solution for the crossover line for the $s$-wave pairing case is presented in Fig.1, where we have put Fermi energy $\varepsilon_{F}$ instead of $n_{f}$, since in the 2D case they are connected by a linear relation $\varepsilon_{F}=\pi n_{f} /\left(m a_{0}^{2}\right)$, and the dimensionless coupling constant $g_{s}=m \lambda_{s} /(4 \pi)$ is introduced. The solution for the $r_{0}\left(n_{f}\right)=$ const case is also presented. As it follows from the numerical calculations, there exists a minimal value of coupling $g_{s}=\simeq 2.574$, which corresponds to carrier density $\varepsilon_{F} \simeq 6.8 \times 10^{-4} W$ on the crossover line, necessary to generate the crossover from superfluidity to superconductivity with doping, otherwise the Cooper pairing regime takes place at any charge carrier density. In other words, there is a minimal value of coupling constant which leads to the two-particle bound states in the $s$-wave pairing channel at small $\varepsilon_{F}$. It is important to note, that there is no such a minimal coupling in the $s$-wave pairing channel when the correlation length $r_{0}\left(n_{f}\right)=$ const [10]. Moreover, the crossover with charge carrier density increasing in the $s$-wave channel takes place for any known doping independent interaction potential 12].

Another interesting property, which follows from the Fig.1b), is the "inverse" crossover from superfluidity to superconductivity with charge carrier density decreasing at small values of $\varepsilon_{F}$ $\left(\varepsilon_{F}<6.8 \times 10^{-4} W\right)$. This is a consequence of the competition between two opposite processes which occur with $\epsilon_{F}$ decreasing. The lowering of $\varepsilon_{F}$ tends the system to become a superfluid, but at the same time it leads to growing of $r_{0}$ and

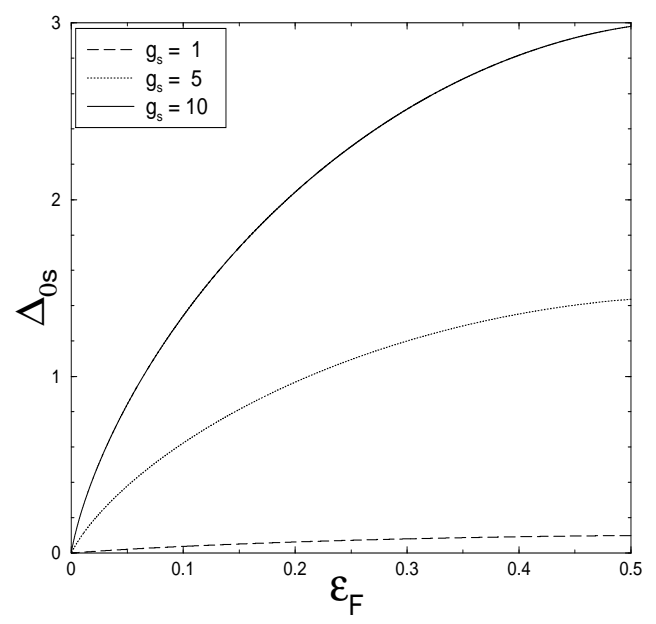

Figure 2. The dependence of $\Delta_{0 s}$ on $\varepsilon_{F}$ at different coupling parameters $g_{s}$ is presented for the $s$-wave case.

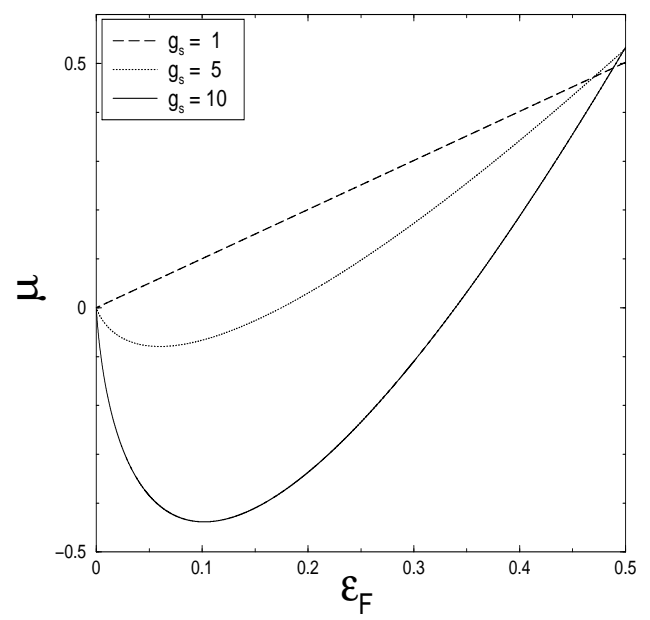

Figure 3. The dependence of $\mu$ on $\varepsilon_{F}$ is presented for the $s$-wave pairing channel at different values of $g_{s}$. 


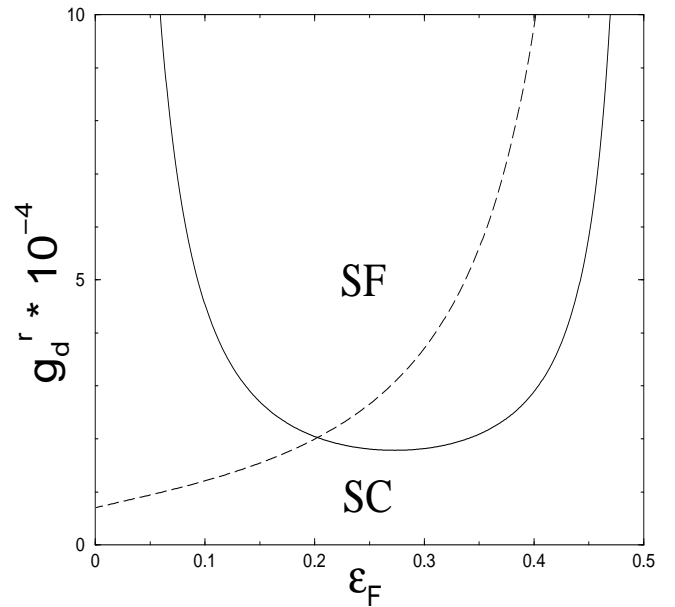

Figure 4 . The crossover line $g_{d}^{r}\left(\varepsilon_{F}\right)$ is presented for the $d$-wave case (solid line). The dotted line is the crossover curve for the case $r_{0}\left(n_{f}\right)=$ const at $r_{0}=a_{0}$.

makes the pair size larger, i.e. the pairs become bounded weaker. On the other hand, the carrier localization on dopants at very small carrier densities also makes a possible density region of superfluidity more narrow 15$]$. However, the region of extremely small particle densities $\varepsilon_{F} \rightarrow 0$ is not very interesting from the point of view of the connection with HTSC, since in this region the relation $r_{0} \sim 1 / \sqrt{n_{f}}$ does not hold, and the model is not correct. As it will be shown in the next Subsection, in the $d$-wave case this crossover from from superfluidity to superconductivity with doping decreasing takes place at rather large Fermi energy values.

The doping dependencies of the gap $\Delta_{0 s}$ and the chemical potential $\mu$ at different values of the coupling constant $g_{s}$ are presented in Figs. 2, and 3. The gap is increasing with the doping almost linearly, except the region of extremely low concentrations. It is interesting to note, that the chemical potential in the superfluidity region $(\mu<0)$ has a minimum at a finite value of $\varepsilon_{F}$ and it is equal to zero at $\varepsilon_{F}=0$, since the effective coupling constant at low doping

$g_{l} \sim k_{0} k_{F}^{2 l} g_{l} \sim \varepsilon_{F}^{l+1 / 2}$

is zero at $\varepsilon_{F}=0$. In other words, the local pairs are the most strongly coupled at some finite carrier density value. This is also a consequence of the competition between the Fermi surface formation and correlation radius decreasing with charge carrier density growth. This situation is qualitatively different from the $r_{0}\left(n_{f}\right)=$ const case, where $k_{0}$ does not depend on particle density and renormalized coupling constant is $\varepsilon_{F}$-independent in the $s$-channel. In this case $\mu=E_{b} / 2<0$ at $\varepsilon_{F}=0$, where $E_{b}<0$ is a two-particle bound state energy.

\section{2. $d$-wave pairing channel}

In this case the Coulomb repulsion parameter $k_{1}$ is present in the equations (7) and (8). However, the presence of this parameter leads just to renormalization of the dimensionless coupling constant $g_{d}=m \lambda_{d} /(4 \pi)$ and the energy gap parameter: $g_{d}^{r}=g_{d} /\left(\varepsilon_{1} / W\right)^{2}, \Delta_{0 d}^{r}=\Delta_{0 d} / \varepsilon_{1}$, where $\varepsilon_{1}=k_{1}^{2} /(2 m)$ is characteristic energy of Coulomb repulsion. We shall consider the case, when the Coulomb repulsion is much smaller than $W$, i.e. this is the case of a large free-fermion bandwidth. Therefore, the coupling constant is assumed to be large: $g_{d}^{r} \sim 10^{4}-10^{5}$ (see Figs. 4-6). The crossover line $g_{d}^{r}\left(\epsilon_{F}\right)$ for the $d$-wave pairing case is presented in Fig.4. Qualitatively, the behavior of the system with doping and coupling changing in the $d$-wave pairing channel is similar to the $s$ wave case. The important difference is that the low carrier density superconducting state exists at rather high values of $\varepsilon_{F}$. Also in this case there is a minimal value of the coupling constant for two-particle bound states $g_{d}^{r} \simeq 1.7856 \times 10^{4}$ at $\varepsilon_{F} \simeq 0.2731 W$. It should be noted that the existence of the large threshold value for the coupling constant in both channels can be a possible answer on the question why the crossover has been not observed in cuprates.

The charge carrier density dependence of $\Delta_{0 d}^{r}$ and $\mu$ at different coupling parameters $g_{d}^{r}$ are presented in Figs.5 and 6 . The superconducting gap $\Delta_{0 d}$ at low charge carrier densities is much smaller than in the $s$-case, due to stronger 
$\varepsilon_{F}$-dependence of effective coupling constant at low carrier densities (10). The magnitude of the order parameter $\Delta_{0 d}^{r}$ starts to grow almost linearly with $\varepsilon_{F}$ increasing when the Fermi energy is larger of some minimal value (Fig.5). This behavior is qualitatively similar to the doping dependence of the gap of cuprates in the underdoped regime. However, because of its simplicity, the model can't describe the decreasing of the gap with charge carrier density increasing at large values of $n_{f}$ HTSCs. For this other properties of the charge carrier interaction in cuprates have to be taken into account (see the last Section).

The small- $\varepsilon_{F}$ superconducting region with $\mu>$ 0 as well as region of superfluidity are rather large in the $d$-wave pairing channel. Also in this case the chemical potential is equal to zero at $\varepsilon_{F}=0$, i.e. there are no two-particle bound states at very low charge carrier densities. It should be noted that the decreasing of the chemical potential with increasing charge carrier density at small $\varepsilon_{F}$ both in $s$ - and $d$-wave pairing cases indicates a negative electronic compressibility. This can be related to increasing of antiferromagnetic correlations at low carrier densities.

\section{CONCLUSION}

Theoretical description of the behavior of superconductor with carrier density changing is an interesting and important problem, in particular, because of its possible association with HTSCs. The microscopic mechanism of the superconductivity in cuprates is not known so far, and the solution of phenomenological models, which take

into account some of the properties of HTSCs, can help to clarify the nature of their unusual behavior, and maybe even help to understand the microscopic mechanism of the HTSC phenomenon.

In this paper the possibility of the crossover from superfluidity to superconductivity with charge carrier density and coupling constant changing in different pairing channels at $T=0$ was studied for a model, which qualitatively takes into account one of the properties of HTSCs, namely, the doping dependence of correlation length $r_{0}$ at low carrier densities. It has been

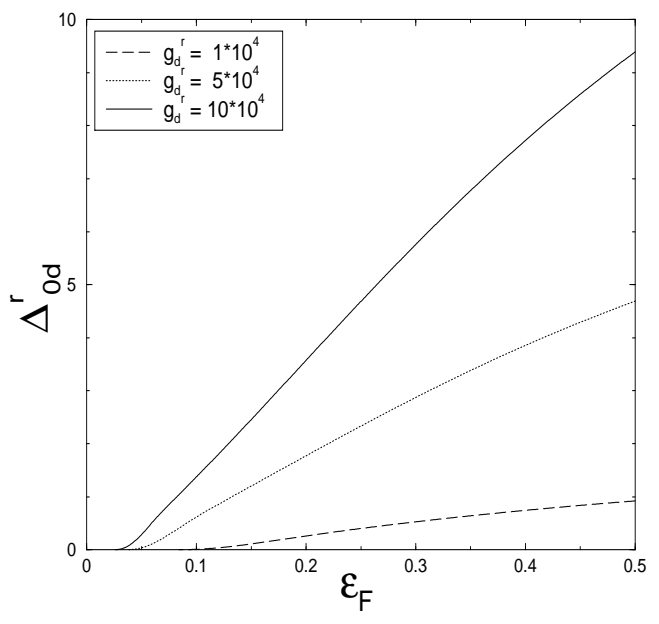

Figure 5. The dependence of $\Delta_{0 d}^{r}$ on $\varepsilon_{F}$ is presented for the $d$-wave pairing channel at different values of coupling constant $g_{d}^{r}$.

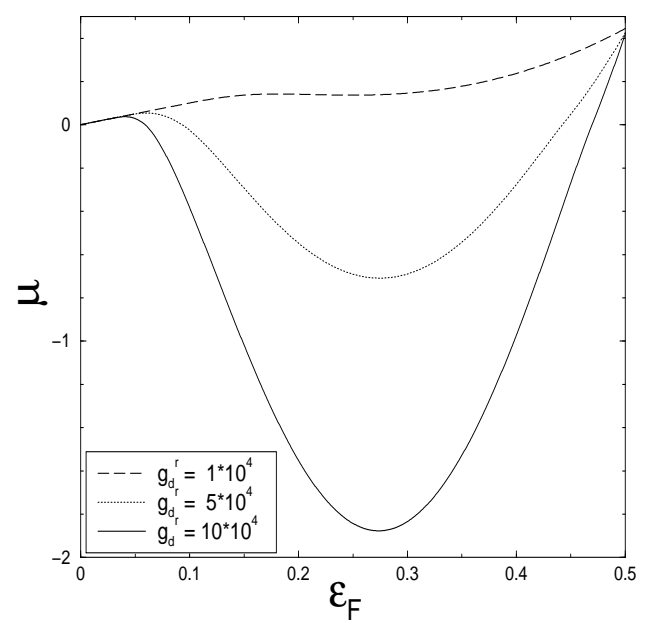

Figure 6. Chemical potential $\mu$ as a function of $\varepsilon_{F}$ is presented for the $d$-wave case at different values of coupling constant $g_{d}^{r}$. 
shown, that even this simple model results in interesting and unusual properties, which are rather different from a more standard case with $r_{0}\left(n_{f}\right)=$ const. In particular, the two-particle bound states in the $s$-channel exist only if the coupling constant is larger of the threshold value, similar to the $d$-wave pairing case. At any value of coupling constant larger of the threshold one, the "inverse" crossover from superfluidity to superconductivity takes place with doping decreasing in both $s$ - and $d$-wave channels. Of course, such a simple model can not pretend to describe doping dependence of the gap and chemical potential of HTSCs. The momentum dependence of the interaction potential has to be taken into account more carefully, especially in the overdoped regime, where $\varepsilon_{F}$ is rather large and the separable potential may be not correct. In general, also the effect of the retardation of interaction can not be neglected. These and some other questions are planed to be studied in a future work.

\section{Acknowledgments}

V.T. thanks the CFIF members, especially Prof. P.D. Sacramento and Prof. V.R. Vieira for kind hospitality. V.M.L. is partly supported by SCOPES-project 7UKPJ062150.00/1 of the Swiss National Science Fundation.

\section{REFERENCES}

1. D.M. Eagles, Phys. Rev. 186 (1969) 456; A.J. Leggett, in: A. Pekalski and J. Przystawa (Eds.), Modern Trends in the Theory of Condensed Matter, Springer-Verlag, Berlin, 1980, p.13; P. Nozieres and S. Schmitt-Rink, J. Low Temp. Phys. 59 (1985) 195.

2. R. Micnas, J. Ranninger, and S. Robaszkiewicz, Rev. Mod. Phys. 62 (1990) 113.

3. M. Randeria, in: A. Griffin, D.W. Snoke, and S. Stringari (Eds.), Bose-Einstein Condensation, Cambridge U.P., New York, 1995, p.355.

4. R. Haussmann, Phys. Rev. B 49 (1994) 12975.

5. E.V. Gorbar, V.M. Loktev, and S.G. Sharapov, Physica C 257 (1996) 355.
6. E.V. Gorbar, V.P. Gusynin, and V.M. Loktev, Sov. Low. Temp. Phys. 19 (1993) 832.

7. V.M. Loktev, V.M. Turkowski, S.G. Sharapov, Teor. i Mat. Fiz. 115 (1998) 419 (English transl.: Teor. and Math. Phys. 115 (1998) $694)$.

8. B.C. den Hertog, Phys. Rev. B 60 (1999) 559.

9. N. Andrenacci, A. Perali, P. Pieri, and G.C. Strinati, Phys. Rev. B 60 (1999) 12410.

10. R.D. Duncan and C.A.R. Sa de Melo, Phys. Rev. B 62 (2000) 9675.

11. N.D. Mermin, H. Wagner, Phys. Rev. Lett. 17 (1966) 1113; P.C. Hogenberg, Phys. Rev. 158 (1967) 383; S. Coleman, Comm. Math. Phys. 31 (1973) 259.

12. V.M. Loktev, R. Quick, S.G. Sharapov, Phys. Rep. 349 (2001) 1.

13. T.R. Thurston, R.J. Birgeneau, M.A. Kastner et al, Phys. Rev. B 40 (1989) 4585.

14. M.Yu. Kagan, T.M.Rice, J. Phys. C: Cond. Matt. 6 (1994) 3771.

15. V.M. Loktev, Yu.G. Pogorelov, Physica C 272 (1996) 151. 